\section{Contribution of In Vivo and In Vitro Testing for The Diagnosis of Local Allergic Rhinitis}

Duarte Ferreira $\mathrm{R}^{1,3}$, Ornelas $\mathrm{C}^{1}$, Silva $\mathrm{S}^{1,3}$, Morgado $\mathrm{R}^{2}$, Pereira $\mathrm{D}^{2}$, Escaleira $\mathrm{D}^{2}$, Moreira $\mathrm{S}^{2}$, Valença $\mathrm{J}^{2}$, Pedro $\mathrm{E}^{1}$, Branco Ferreira $\mathrm{M}^{1,4}$, Conceição Pereira Santos $\mathrm{M}^{3,4}$, Barbosa $\mathrm{M}^{1,4}$

${ }^{I}$ Serviço de Imunoalergologia, Centro Hospitalar de Lisboa Norte, Lisbon, Portugal

${ }^{2}$ Laboratório de Fisiopatologia Respiratória, Serviço de Pneumologia, Centro Hospitalar de Lisboa Norte, Lisbon, Portugal

${ }^{3}$ Laboratório de Imunologia Clínica, Instituto de Medicina Molecular, Faculdade de Medicina da Universidade de Lisboa, Lisbon, Portugal

${ }^{4}$ Clínica Universitária de Imunoalergologia, Faculdade de Medicina da Universidade de Lisboa, Lisbon, Portugal

J Investig Allergol Clin Immunol 2019; Vol. 29(1): 46-48 doi: 10.18176/jiaci.0321

Key words: Local allergic rhinitis. Basophil activation test. Allergy diagnosis. Rhinomanometry.

Palabras clave: Rinitis alérgica local. Test de activación de basófilos. Diagnóstico alérgico. Rinomanometría.

Local allergic rhinitis (LAR) is characterized by an allergen-specific, IgE-mediated inflammatory response limited to the nasal mucosa [1]. This local production of IgE (entopy) is not associated with measurable specific $\operatorname{IgE}(\mathrm{s} \operatorname{IgE})$ in peripheral blood or with positivity in skin prick testing (SPT) for the culprit allergen. In southern Europe, LAR is estimated to account for $26 \%$ of all cases of rhinitis and up to $70 \%$ of all cases of nonallergic rhinitis (NAR) [2]. Despite similar disease mechanisms, LAR seems to be distinct from classic allergic rhinitis and not its precursor [3].

LAR should be suspected when the patient's symptoms are suggestive and triggered by allergen exposure, despite negative findings in a conventional allergological work-up. Patients are frequently misdiagnosed with idiopathic NAR, and distinguishing between both entities is difficult without specific diagnostic tests, which may not be available in all centers [4]. Underdiagnosis of LAR has implications for the management of these patients, as they are deprived of allergen immunotherapy (AIT), an effective approach in such cases [5].

The nasal provocation test (NPT) is considered the goldstandard for diagnosis, although it can be time-consuming [6]. Given the limited experience using the basophil activation test (BAT) as part of a comprehensive diagnostic approach to LAR $[7,8]$, we re-evaluated the relative contribution of this test in patients with suspected LAR and determined whether it would help to avoid in vivo testing.

We report data from 20 patients with rhinitis symptoms triggered by exposure to house dust mites (mean and median age, 45.5 years; $80 \%$ female; mean age at time of diagnosis, 31 years; median age, 30.2 years). We also evaluated 4 healthy controls and 4 allergic rhinitis patients (mean age, 33.4 years; median age, 31.5 years).

All patients had negative SPT results and SIgE for dust mites - as did the healthy controls - and underwent NPT with a dust mite extract (Laboratorios LETI). An extract of Dermatophagoides pteronyssinus $(100 \mathrm{HEP} / \mathrm{mL})$ or Lepidoglyphus destructor $(30 \mathrm{HEP} / \mathrm{mL})$ was selected according to the patient's clinical history and/or occupational exposure. The provocation test was performed with $1 / 1000$, $1 / 100$, and $1 / 10$ dilutions of mite extract applied nasally using a metered pump at 15 -minute intervals. The result of the NPT was determined using clinical and/or rhinomanometric criteria. The clinical D pteronyssinus criteria included triggered symptoms, such as sneezing, nasal pruritus, obstruction, anterior or posterior rhinorrhea, and tearing. A visual analog scale was used for each symptom, and an increase $>30 \%$ for the sum of all 5 parameters was considered positive [6]. A baseline and post-NPT rhinomanometric evaluation was performed (MasterScreen Body, Jaeger), and a 50\% increase in resistance was considered positive [9].

sIgE was quantified in nasal secretions before the NPT (T0), at the end of the NPT (T1), and 1 hour later (T2) (ImmunoCAP, Thermo Fisher Scientific). A BAT (Buhlmann) was performed according to the manufacturer's instructions with mite extracts (LETI, see above) in 17 of the 20 patients and in the controls. The $D$ pteronyssinus extract was run with the commercially available concentration of $1.19 \mathrm{mg} / \mathrm{mL}(100 \mathrm{HEP} / \mathrm{mL})$ and with concentrations of $0.595,0.298,0.0595,0.029$, and $0.019 \mathrm{mg} / \mathrm{mL}$. For $L$ destructor, the BAT was performed with the commercially available concentration of $5 \mathrm{mg} / \mathrm{mL}(30 \mathrm{HEP} / \mathrm{mL})$ and with concentrations of $1.4,0.35$, and $0.025 \mathrm{mg} / \mathrm{mL}$. Basophils were identified as $\mathrm{CCR} 3^{+}$cells, and CD63 expression was used as a marker of basophil activation. The study was approved by the local ethics committee. Informed consent was obtained from all participants.

Seventeen of the 20 NPTs ( $85 \%$ ) were positive. Fourteen NPTs were performed with $D$ pteronyssinus (13 positive) and 6 with $L$ destructor (4 positive). Four NPTs were positive only by clinical criteria, 7 only by rhinomanometric criteria, and another 6 by both (Table). There were no significant differences in the quantification of $\operatorname{sIgE}(\mathrm{kU} / \mathrm{L})$ in nasal secretions between patients with positive or negative NPT results, or between the different collection times of nasal secretions (T0, median [IQR], 0.125 [0.113-0.138], mean, 0.130; T1, median, 0.130 [0.110-0.130], mean, 0.125; T2, median, 0.125 [0.113-0.130], mean, 0.120).

The BAT was performed in 17 patients, of whom 15 had positive NPT results. The results were all negative for healthy 
Table. Nasal Provocation Test and Basophil Activation Test Results

\begin{tabular}{|c|c|c|c|c|c|c|}
\hline Subject & Extract & NPT Result ${ }^{\mathrm{a}}$ & $\begin{array}{c}\Delta \mathrm{VAS}>30 \%, \\
\mathrm{~cm}\end{array}$ & $\begin{array}{c}\text { Rhinomanometry } \\
\Delta \text { Res } \geq 50 \%\end{array}$ & $\begin{array}{l}\text { Nasal sIgE, kU/L } \\
\quad(\mathrm{T} 0, \mathrm{~T} 1, \mathrm{~T} 2)\end{array}$ & $\begin{array}{l}\text { BAT results } \\
\text { (SI) }\end{array}$ \\
\hline $\mathrm{F}, 60$ & LD & $\begin{array}{c}+ \\
(0.50)\end{array}$ & $\begin{array}{c}\text { Yes } \\
(7.2 \rightarrow 15.7)\end{array}$ & $\begin{array}{c}\text { Yes } \\
(152 \% \rightarrow 289 \%)\end{array}$ & $0.11,0.11,0.11$ & $\begin{array}{c}+ \\
(45.86)\end{array}$ \\
\hline F, 29 & DP & $\begin{array}{c}+ \\
(0.12)\end{array}$ & $\begin{array}{l}\text { Yes } \\
(3.7 \rightarrow 12.7)\end{array}$ & $\begin{array}{c}\text { Yes } \\
(151 \% \rightarrow 268 \%)\end{array}$ & $0.12,0.13,0.12$ & $\begin{array}{l}- \\
\text { (1) }\end{array}$ \\
\hline F, 39 & DP & $\begin{array}{c}+ \\
(0.01)\end{array}$ & $\begin{array}{c}\text { Yes } \\
(1.7 \rightarrow 3.3)\end{array}$ & $\begin{array}{c}\text { Yes } \\
(50 \% \rightarrow 170 \%)\end{array}$ & $0.13,0.13,0.13$ & $\begin{array}{c}+ \\
(6.49)\end{array}$ \\
\hline M, 46 & DP & $\begin{array}{c}+ \\
(0.12)\end{array}$ & $\begin{array}{c}\text { Yes } \\
(3.1 \rightarrow 6.2)\end{array}$ & $\begin{array}{c}\text { Yes } \\
(77 \% \rightarrow 187 \%)\end{array}$ & $0.13,0.13,0.12$ & $(0.73)$ \\
\hline $\mathrm{F}, 47$ & DP & $\begin{array}{c}+ \\
(0.12)\end{array}$ & $\begin{array}{c}\text { Yes } \\
(3.6 \rightarrow 4.8)\end{array}$ & $\begin{array}{c}\text { Yes } \\
(132 \% \rightarrow 235 \%)\end{array}$ & $0.13,0.13,0.13$ & $\begin{array}{l}- \\
(0)\end{array}$ \\
\hline $\mathrm{F}, 54$ & DP & $\begin{array}{c}+ \\
(0.12)\end{array}$ & $\begin{array}{c}\text { Yes } \\
(0 \rightarrow 28.3)\end{array}$ & $\begin{array}{c}\text { Yes } \\
(51 \% \rightarrow 139 \%)\end{array}$ & $0.14,0.13,0.12$ & $\begin{array}{c}+ \\
(3.39)\end{array}$ \\
\hline $\mathrm{F}, 35$ & LD & $\begin{array}{c}+ \\
(0.05)\end{array}$ & $\begin{array}{c}\text { Yes } \\
(22.6 \rightarrow 30.9)\end{array}$ & $\begin{array}{c}\text { No } \\
(62 \% \rightarrow 102 \%)\end{array}$ & $0.11,0.11,0.12$ & $\begin{array}{c}+ \\
(5.44)\end{array}$ \\
\hline $\mathrm{F}, 27$ & DP & $\begin{array}{c}+ \\
(0.12)\end{array}$ & $\begin{array}{c}\text { Yes } \\
(5.7 \rightarrow 14)\end{array}$ & $\underset{(130 \% \rightarrow 149 \%)}{\text { No }}$ & $0.22,0.15,0.13$ & NP \\
\hline $\mathrm{F}, 40$ & $\mathrm{DP}$ & $\begin{array}{c}+ \\
(0.12)\end{array}$ & $\begin{array}{l}\text { Yes } \\
(0 \rightarrow 8)\end{array}$ & $\begin{array}{c}\text { No } \\
(75 \% \rightarrow 102 \%)\end{array}$ & $0.16,0.15,0.14$ & $\begin{array}{c}- \\
(0)\end{array}$ \\
\hline M, 43 & DP & $\begin{array}{c}+ \\
(0.12)\end{array}$ & $\begin{array}{c}\text { Yes } \\
(3.3 \rightarrow 6.3)\end{array}$ & $\begin{array}{c}\text { No } \\
(45 \% \rightarrow 70 \%)\end{array}$ & $0.12,0.13,0.13$ & $\begin{array}{c}+ \\
(2.95)\end{array}$ \\
\hline F, 53 & LD & $\begin{array}{l}+ \\
(0.5)\end{array}$ & $\begin{array}{c}\text { No } \\
(20 \rightarrow 20.4)\end{array}$ & $\begin{array}{c}\text { Yes } \\
(63 \% \rightarrow 117 \%)\end{array}$ & $0.12,0.11,0.10$ & $\begin{array}{c}+ \\
(9.72)\end{array}$ \\
\hline $\mathrm{F}, 64$ & LD & $\begin{array}{l}+ \\
(0.5)\end{array}$ & $\begin{array}{c}\mathrm{No} \\
(3.3 \rightarrow 4.1)\end{array}$ & $\underset{(44 \% \rightarrow 116 \%)}{\text { Yes }}$ & $0.10,0.11,0.10$ & $\begin{array}{c}+ \\
(82.88)\end{array}$ \\
\hline $\mathrm{F}, 22$ & DP & $\begin{array}{l}+ \\
(0.12)\end{array}$ & $\begin{array}{c}\text { No } \\
(7.9 \rightarrow 6.6)\end{array}$ & $\begin{array}{c}\text { Yes } \\
(69 \% \rightarrow 119 \%)\end{array}$ & $0.15,0.15,0.15$ & $\mathrm{NP}$ \\
\hline F, 43 & DP & $\begin{array}{c}+ \\
(0.12)\end{array}$ & $\begin{array}{c}\text { No } \\
(3.7 \rightarrow 1.9)\end{array}$ & $\begin{array}{c}\text { Yes } \\
(65 \% \rightarrow 214 \%)\end{array}$ & $0.12,0.13,0.14$ & $(1.65)$ \\
\hline $\mathrm{F}, 47$ & DP & $\begin{array}{c}+ \\
(0.12)\end{array}$ & $\begin{array}{c}\text { No } \\
(0.7 \rightarrow 0)\end{array}$ & $\begin{array}{c}\text { Yes } \\
(110 \% \rightarrow 229 \%)\end{array}$ & $0.13,0.11,0.13$ & $\begin{array}{l}- \\
(0)\end{array}$ \\
\hline F, 55 & DP & $\begin{array}{l}+ \\
(0.12)\end{array}$ & $\begin{array}{l}\text { No } \\
(0 \rightarrow 0)\end{array}$ & $\underset{(58 \% \rightarrow 255 \%)}{\text { Yes }}$ & $0.13,0.13,0.13$ & $\begin{array}{c}+ \\
(2.30)\end{array}$ \\
\hline F, 58 & DP & $\begin{array}{c}+ \\
(0.12)\end{array}$ & $\begin{array}{c}\text { No } \\
(33.2 \rightarrow 1.5)\end{array}$ & $\begin{array}{c}\text { Yes } \\
(114 \% \rightarrow 245 \%)\end{array}$ & $0.12,0.12,0.13$ & NR \\
\hline F, 43 & LD & - & $\begin{array}{c}\text { No } \\
(1.8 \rightarrow 2)\end{array}$ & $\begin{array}{c}\text { No } \\
(48 \% \rightarrow 69 \%)\end{array}$ & $0.10,0.11,0.10$ & $\begin{array}{l}- \\
(0)\end{array}$ \\
\hline $\mathrm{F}, 45$ & LD & - & $\begin{array}{c}\text { No } \\
(11.6 \rightarrow 14)\end{array}$ & $\begin{array}{c}\text { No } \\
(69 \% \rightarrow 83 \%)\end{array}$ & $0.11,0.10,0.10$ & NP \\
\hline $\mathrm{F}, 60$ & DP & - & $\begin{array}{c}\text { No } \\
(1 \rightarrow 0)\end{array}$ & $\begin{array}{c}\text { No } \\
(298 \% \rightarrow 180 \%)\end{array}$ & $0.14,0.12,0.12$ & $\overline{(0)}$ \\
\hline \multicolumn{7}{|c|}{ Allergic (Positive) Controls } \\
\hline $\mathrm{M}, 18$ & DP & $\begin{array}{c}+ \\
(0.01)\end{array}$ & $\begin{array}{c}\text { Yes } \\
(6.1 \rightarrow 16.8)\end{array}$ & $\begin{array}{c}\text { Yes } \\
(149 \% \rightarrow 277 \%)\end{array}$ & $0.13,0.12,0.14$ & $\begin{array}{c}+ \\
(33.62)\end{array}$ \\
\hline $\mathrm{F}, 41$ & DP & $\begin{array}{l}+ \\
(0.12)\end{array}$ & $\begin{array}{c}\text { Yes } \\
(5.2 \rightarrow 17.7)\end{array}$ & $\begin{array}{c}\text { Yes } \\
(124 \% \rightarrow 292 \%)\end{array}$ & $0.11,0.11,0.12$ & $\begin{array}{c}+ \\
(66.37)\end{array}$ \\
\hline F, 26 & LD & $\begin{array}{c}+ \\
(0.5)\end{array}$ & $\begin{array}{c}\text { Yes } \\
(0 \rightarrow 9.1)\end{array}$ & $\underset{(71 \% \rightarrow 134 \%)}{\text { Yes }}$ & $0.12,0.13,0.13$ & $\begin{array}{c}+ \\
(58.09)\end{array}$ \\
\hline $\mathrm{F}, 35$ & LD & $\begin{array}{l}+ \\
(0.5)\end{array}$ & $\begin{array}{c}\text { No } \\
(16 \rightarrow 16.7)\end{array}$ & $\begin{array}{c}\text { Yes } \\
(67 \% \rightarrow 205 \%)\end{array}$ & $0.10,0.12,0.14$ & $\begin{array}{c}+ \\
(87.57)\end{array}$ \\
\hline \multicolumn{7}{|c|}{ Healthy (Negative) Controls } \\
\hline M, 20 & $\mathrm{DP}$ & - & $\begin{array}{c}\text { No } \\
(3 \rightarrow 2.5)\end{array}$ & $\begin{array}{c}\text { No } \\
(53 \% \rightarrow 88 \%)\end{array}$ & $0.10,0.10,0.10$ & $\begin{array}{c}- \\
(0)\end{array}$ \\
\hline M, 47 & $\mathrm{DP}$ & - & $\stackrel{\text { No }}{(11 \rightarrow 12.7)}$ & $\begin{array}{c}\text { No } \\
(61 \% \rightarrow 89 \%)\end{array}$ & $0.11,0.11,0.10$ & $\begin{array}{l}- \\
(0)\end{array}$ \\
\hline $\mathrm{F}, 28$ & LD & - & $\begin{array}{l}\text { No } \\
(0 \rightarrow 0)\end{array}$ & $\begin{array}{c}\text { No } \\
(158 \% \rightarrow 180 \%)\end{array}$ & $0.11,0.12,0.14$ & - \\
\hline F, 52 & LD & - & $\begin{array}{l}\mathrm{No} \\
(1 \rightarrow 0)\end{array}$ & $\begin{array}{c}\text { No } \\
(104 \% \rightarrow 99 \%)\end{array}$ & $0.10,0.12,0.12$ & $\begin{array}{l}- \\
(0)\end{array}$ \\
\hline
\end{tabular}

Abbreviations: BAT, basophil activation test; DP, Dermatophagoides pteronyssinus; LD, Lepidoglyphus destructor; NP, not performed; NR, nonresponder; SI, stimulation index; $\Delta$ Res, variation in measured resistance; $\Delta$ VAS, variation in the result of the visual analog scale.

${ }^{a}$ Allergen concentration with positive response in $\mathrm{mg} / \mathrm{mL}$. 
controls and all positive for allergic rhinitis controls. The BAT was positive (stimulation index, $\geq 2$ ) in 8 of the 15 patients with a positive NPT result (53.3\%) and negative in 8 patients, 2 of whom had a negative NPT result. One patient was a nonresponder. In 6 patients with a positive NPT result, the result of the BAT was negative.

Despite the small sample, a diagnosis of LAR (positive NPT result) was established in $85 \%$ of participants. This large percentage emphasizes the importance of further investigation in these patients, for whom current evidence supports treatment with AIT. In one study, AIT improved symptoms and increased allergen tolerance, as measured by NPT [5].

The positivity criteria for NPT varied in the literature until the recent publication of unified guidelines [10]. Clinical symptoms are insufficient for interpreting NPT results, as further evidenced in our study, where $41.2 \%$ of NPTs were considered positive solely by rhinomanometric criteria.

We observed no significant variation in sIgE levels. Determination of SIgE in nasal secretions is very specific, although less sensitive than NPT, probably because of a dilution effect of nasal lavage [4].

In LAR, BAT has been shown to have a sensitivity of $50.0 \%-66.6 \%$ and a specificity of $90.0 \%-91.7 \%[7,8]$. In our study, BAT and NPT results agreed in 10 out of 16 patients $(62.5 \%)$. There were no false positives. The sensitivity of BAT obtained in the present study $(53.3 \%)$ reproduces the results of previous studies $[7,8]$ and reinforces the usefulness of BAT in the first steps of a rational diagnostic approach in LAR and when NPT is not available.

NPT is a time-consuming procedure. Our findings and the high specificity of BAT obtained in previous studies performed in LAR patients suggest that a positive BAT result could confirm a diagnosis of LAR. We propose that, where possible, the various available diagnostic methods should be combined to maximize diagnostic accuracy.

\section{Funding}

Laboratorios LETI provided funding for the nasal provocation kits.

\section{Conflicts of Interest}

The authors declare that they have no conflicts of interest.

\section{References}

1. Rondón C, Fernandez J, Canto G, Blanca M. Local allergic rhinitis: Concept, clinical manifestations, and diagnostic approach. J Investig Allergol Clin Immunol. 2010;20(5):36471.

2. Rondón C, Campo P, Galindo L, Blanca-López N, Cassinello MS, Rodriguez-Bada $J$, et al. Prevalence and clinical relevance of local allergic rhinitis. Allergy [Internet]. 2012;67(10):1282-8. Available from: http://www.ncbi.nlm. nih.gov/pubmed/22913574

3. Campo P, Rondón C, Gould HJ, Barrionuevo E, Gevaert P, Blanca M. Local IgE in non-allergic rhinitis. Clin Exp Allergy. 2015;45(5):872-81.
4. Campo P, Salas M, Blanca-López N, Rondón C. Local Allergic Rhinitis. Immunol Allergy Clin North Am [Internet]. 2016;36(2):321-32. Available from: http://dx.doi. org/10.1016/j.iac.2015.12.008

5. Rondón C, Blanca-López N, Campo P, Mayorga C, JuradoEscobar R, Torres MJ, et al. Specific immunotherapy in local allergic rhinitis: A randomized, double-blind placebocontrolled trial with Phleum pratense subcutaneous allergen immunotherapy. Allergy Eur J Allergy Clin Immunol. 2018;73(4):905-15.

6. Rondón C, Campo P, Herrera R, Blanca-Lopez N, Melendez $L$, Canto G, et al. Nasal allergen provocation test with multiple aeroallergens detects polysensitization in local allergic rhinitis. J Allergy Clin Immunol [Internet]. 2011 Dec;128(6):1192-7. Available from: http://www.ncbi.nlm.nih. gov/pubmed/21783237

7. Gómez E, Campo P, Rondón C, Barrionuevo E, Blanca-López $\mathrm{N}$, Torres MJ, et al. Role of the basophil activation test in the diagnosis of local allergic rhinitis. J Allergy Clin Immunol. 2013;132(4).

8. Campo P, Villalba M, Barrionuevo E, Rondón C, Salas M, Galindo $L$, et al. Immunologic responses to the major allergen of Olea europaea in local and systemic allergic rhinitis subjects. Clin Exp Allergy. 2015;45(11):1703-12.

9. Pirilä T, Nuutinen J. Acoustic rhinometry, rhinomanometry and the amount of nasal secretion in the clinical monitoring of the nasal provocation test. Clin Exp Allergy. 1998;28(4):468-77.

10. Augé J, Vent J, Agache I, Airaksinen L, Campo Mozo P, Chaker $A$, et al. EAACI Position paper on the standardization of nasal allergen challenges. Allergy [Internet]. 2018 Aug;73(8):1597608. Available from: http://doi.wiley.com/10.1111/all.13416

- Manuscript received May 21, 2018; accepted for publication September 12, 2018.

Ruben Duarte Ferreira

Hospital de Santa Maria Avenida Prof. Egas Moniz, 1649-035 Lisboa, Portugal E-mail: ruben.ferreira@gmail.com 